\title{
Correction to: MoMyb1 is required for asexual development and tissue-specific infection in the rice blast fungus Magnaporthe oryzae
}

Yanhan Dong ${ }^{1,2}$, Qian Zhao, Xinyu Liu ${ }^{1,2}$, Xiaofang Zhang ${ }^{1,2}$, Zhongqiang Qi $i^{1,2}$, Haifeng Zhang ${ }^{1,2^{*}}$, Xiaobo Zheng ${ }^{1,2}$ and Zhengguang Zhang ${ }^{1,2}$

\section{Correction}

Following the publication of this article [1], the authors noticed that they mistakenly introduced duplicate images in Fig. 6a during the preparation of figures. They apologize for any confusion that brought to the readers and have corrected the figure. This correction does not change any statement or conclusion drawn from the data.

The correct version of Fig. 6a has been included in this correction.

Received: 18 September 2018 Accepted: 18 September 2018

Published online: 03 October 2018

\section{Reference}

1. Dong $Y$, et al. MoMyb1 is required for asexual development and tissue-

specific infection in the rice blast fungus Magnaporthe oryzae. BMC

Microbiol. 2015;15:37. https://doi.org/10.1186/s12866-015-0375-y.

\footnotetext{
* Correspondence: hfzhang@njau.edu.cn

'Department of Plant Pathology, College of Plant Protection, Nanjing Agricultural University, Nanjing 210095, China

${ }^{2}$ Key Laboratory of Integrated Management of Crop Diseases and Pests, Ministry of Education, Nanjing 210095, China
} 


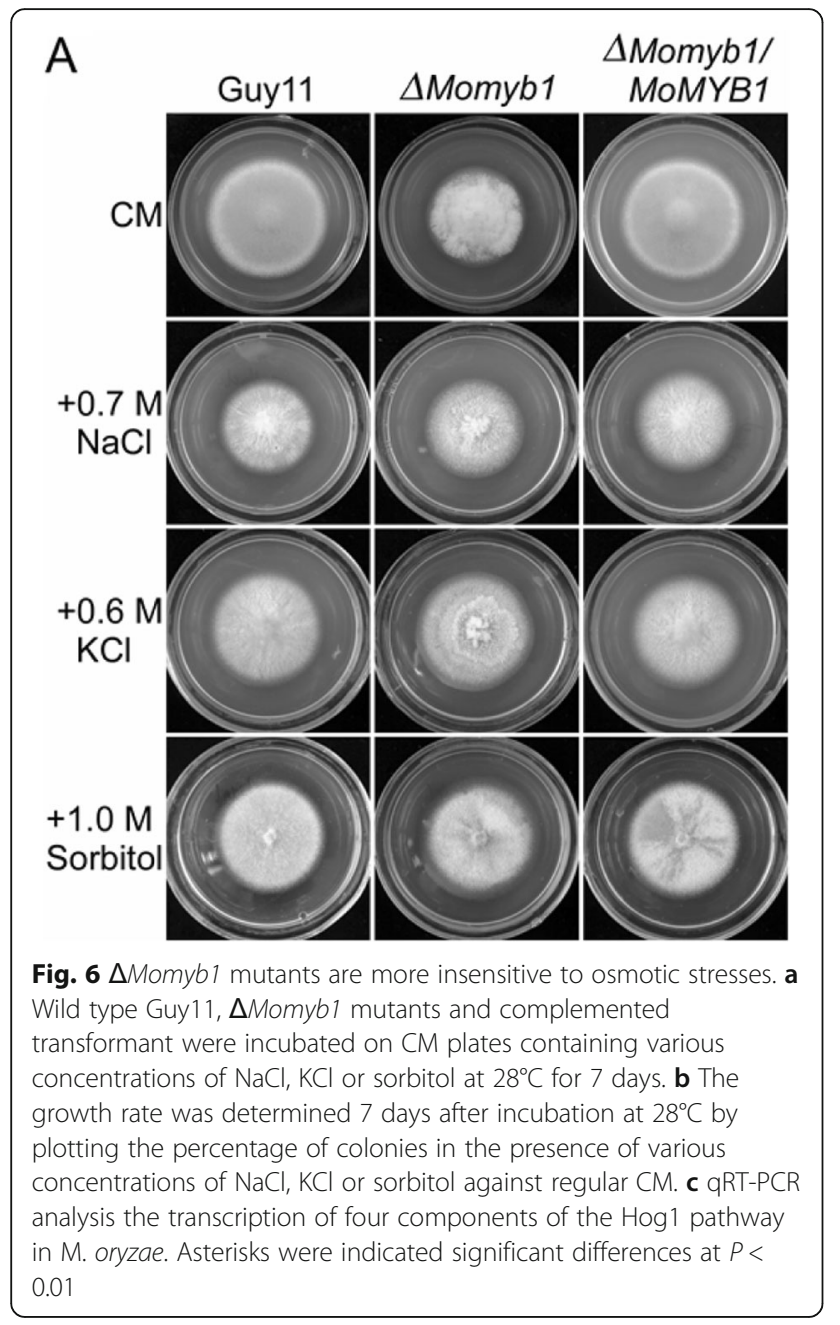

